Original Article

\title{
Outcomes of Concomitant Total Aortic Arch Replacement with Coronary Artery Bypass Grafting
}

Ken Yamanaka, MD, Tatsuhiko Komiya, MD, Hiroshi Tsuneyoshi, MD, PhD, and Takeshi Shimamoto, MD, PhD

\begin{abstract}
Objective: Total aortic arch replacement is a highly invasive procedure. Here, we have investigated patient outcomes following total aortic arch replacement with or without coronary artery bypass grafting.

Methods: One hundred and eighty-one patients underwent total aortic arch replacement without coronary artery bypass grafting, and 65 underwent with coronary artery bypass grafting. We compared preoperative, operative, and postoperative factors and analyzed survival outcomes. We used univariate and multivariate analyses to determine factors associated with long-term mortality.

Results: Cardiopulmonary bypass and surgical times were significantly longer in the concomitant total aortic arch replacement with coronary artery bypass grafting group. Hospital mortality was $3.3 \%$ in the total aortic arch replacement group and $7.7 \%$ in the concomitant total aortic arch replacement with coronary artery bypass grafting group. Perioperative myocardial infarction was not seen in either group. There were no significant differences in mortality between the groups. Multivariate analysis revealed preoperative age, ischemic heart disease, and estimated glemerular filtration rate (eGFR) as risk factors affecting long-term mortality, whereas concomitant total aortic arch replacement with coronary artery bypass grafting was not a risk factor.

Conclusion: Although patients' backgrounds should be considered, total aortic arch replacement can be concomitantly performed with coronary artery bypass grafting surgery without additional mortality risk.
\end{abstract}

Keywords: aortic surgery, coronary artery bypass grafting, total arch replacement

\section{Introduction}

Surgical techniques and postoperative intensive care have markedly advanced in recent times. Overall hospital

Department of Cardiovascular Surgery, Kurashiki Central Hospital, Kurashiki, Okayama, Japan

Received: February 23, 2016; Accepted: April 20, 2016

Corresponding author: Ken Yamanaka, MD. Department of Cardiovascular Surgery, Kurashiki Central Hospital, 1-1-1 Miwa, Kurashiki, Okayama 710-8602, Japan

Email: yama.ken5.21@gmail.com

(C2016 The Editorial Committee of Annals of Thoracic and

Cardiovascular Surgery. All rights reserved. mortality after total aortic arch replacement (TAR) has been declining, ${ }^{1-3)}$ but TAR, which requires hypothermia and prolonged cardiopulmonary bypass, remains a highly invasive procedure. Subgroups exist that may be at a surgical risk due to preoperative comorbidity, particularly patients with significant coronary artery stenosis. Some reports have suggested that a higher risk is associated with performing coronary artery bypass grafting (CABG) and TAR concomitantly. ${ }^{4-5}$ ) Other recent studies have reported that concomitant $\mathrm{CABG}$ did not increase mortality and morbidity. ${ }^{6-7)}$ The aim of this study was to investigate patient outcomes following TAR performed with or without CABG. 


\section{Patients and Methods}

From January 2002 to December 2014, a total of 246 patients underwent elective TAR with or without CABG under hypothermic arrest of the lower body via a median sternotomy. Patients who underwent ascending aortic surgery without arch reconstruction, descending aortic surgery, emergent surgery, and stent grafting cases were excluded from this analysis. We divided 246 patients into two groups according to whether they received TAR with $\mathrm{CABG}$ (TAR/CABG; $\mathrm{n}=65$ ) or without $\mathrm{CABG}$ (TAR; $\mathrm{n}=181$ ).

Study patients were followed at the outpatient clinic, patients' family physicians or by telephone contact. The follow up rate was $91.8 \%$. The early and midterm clinical outcomes were analyzed.

\section{Surgical technique}

General anesthesia was achieved using standard techniques. In all patients, the axillary artery was exposed with a deltopectoral groove incision and all procedures were performed using a median sternotomy. The internal thoracic artery (ITA) grafts were harvested using a skeletonized technique and were used as in-situ grafts whenever possible. The saphenous vein (SV) or radial artery was harvested using an open method.

The ascending aorta was cannulated for systemic flow and the right atrial appendage was cannulated with a twostage cannula for systemic venous return. If the ascending aorta had severe atherosclerotic change, the subclavian artery or the femoral artery was cannulated for systemic flow. The patients were placed on cardiopulmonary bypass and underwent systemic cooling to $28^{\circ} \mathrm{C}$. During this period, the right axillary artery was cannulated with a 12 Fr cannula. If CABG was necessary, we harvested the artery graft or vein graft when surgery started. On-pump CABG distal anastomosis was performed during systemic cooling. When the deep body temperature decreased to $28^{\circ} \mathrm{C}$, a cross clamp was placed across the ascending aorta and myocardial arrest was achieved with cold blood cardioplegia. After antegrade cardioplegia, we stopped the cardio-pulmonary bypass (CPB) by selective antegrade cerebral perfusion (SACP). When other concomitant procedures were needed, we performed the procedures during systemic cooling. We performed open distal anastomosis in all cases. During this time, we preferred to perform turn-up anastomosis as previously reported by our institution. ${ }^{8)}$ As soon as the distal anastomosis was completed, systemic perfusion from the graft was started. We sequentially reconstructed the left subclavian artery, left common carotid artery, proximal root, and right brachiocephalic artery. Finally, in cases where a free coronary bypass graft was required, the proximal anastomosis was completed. Where it was possible to bypass the native ascending aortic wall, we sutured the free graft to the native aorta, and if not, to the graft.

\section{Definitions of complications}

In-hospital death was defined as death occurring during hospitalization or after discharge from the hospital but within 30 days of the procedure. A postoperative stroke was defined as the rapid onset of neurological deficits and was confirmed by a computed tomography scan. Stroke was defined as the presence of permanent deficits persisting at discharge. The requirement for unexpected $\mathrm{CABG}$ during surgery or the presence of new $\mathrm{Q}$ wave on electrocardiograms was defined as perioperative myocardial infarction (PMI). Major adverse cardiovascular events (MACE) included sudden death from cardiac problems, unknown cause of death, occurrence of a myocardial ischemic event or congestive heart failure.

\section{Statistical analysis}

In this report, data were processed using SPSS 20.0 software (SPSS, Chicago, IL, USA). Data were analyzed by the $\mathrm{X}^{2}$ test for categorical variables. Continuous values were presented as the mean \pm standard deviation. Stepwise logistic regression analysis was performed to identify the risk factors for in-hospital mortality. Clinically relevant variables with a p-value of less than 0.1 on univariate analysis were incorporated into multivariate models. Survival was assessed by the Kaplan-Meier method, and a log rank test was applied for comparison. A Cox proportional hazard analysis was used to determine risk factors for late mortality. Differences were considered statistically significant if the p-value was less than 0.05 .

\section{Results}

We performed CABG in patients with symptoms (e.g., chest pain) or significant stenosis of more than 50\% stenosis of the left main trunk and more than $75 \%$ stenosis of the left anterior descending artery (LAD), circumflex (Cx) artery, right coronary artery (RCA), and key coronary branches that broadly cover the myocardium.

Table 1 shows the preoperative characteristics. The mean age was $71.2 \pm 10.7$ years in the TAR group and 73.1 \pm 6.6 in the TAR/CABG group. The incidence of old myocardial infarction (OMI) and arteriosclerosis obliterans 
Table 1 Preoperative characteristics of the two groups

\begin{tabular}{lccc}
\hline & TAR & TAR/CABG & $\mathrm{p}$ \\
\hline Age & $71.2 \pm 10.7$ & $73.1 \pm 6.6$ & 0.21 \\
Sex (Male) & $76.1 \%$ & $80.3 \%$ & 0.50 \\
BMI & 23.2 & 24.0 & 0.44 \\
DM & $13.9 \%$ & $15.1 \%$ & 0.83 \\
HT & $87.4 \%$ & $92.9 \%$ & 0.38 \\
HL & $39.2 \%$ & $44.6 \%$ & 0.45 \\
eGFR & $60.6 \pm 24.0$ & $54.9 \pm 21.1$ & 0.64 \\
HD & $2.9 \%$ & $0 \%$ & 0.33 \\
ASO & $4.4 \%$ & $12.1 \%$ & 0.02 \\
OMI & $7.1 \%$ & $18.1 \%$ & 0.01 \\
Stroke & $12.2 \%$ & $16.6 \%$ & 0.40 \\
COPD & $9.4 \%$ & $6.1 \%$ & 0.45 \\
Smoking & $71.9 \%$ & $78.7 \%$ & 0.32 \\
EF $<40 \%$ & $6.1 \%$ & $12.1 \%$ & 0.12 \\
Preoperative PCI & 11.0 & $16.9 \%$ & 0.22 \\
LMT disease & 0 & $4.6 \%$ & $<0.01$ \\
1 vessel disease & $9.9 \%$ & $44.6 \%$ & $<0.01$ \\
2 vessel disease & $2.2 \%$ & $30.7 \%$ & $<0.01$ \\
3 vessel disease & $0.55 \%$ & $16.9 \%$ & $<0.01$ \\
Chest pain & 0 & $9.2 \%$ & $<0.01$ \\
Heart failure & 0 & $4.6 \%$ & $<0.01$ \\
\hline
\end{tabular}

TAR: total aortic arch replacement; CABG: coronary artery bypass grafting; BMI: body mass index; DM: diabetes mellitus; HT: hypertension; HL: hyperlipemia; eGFR: estimated glemerular filtration rate; HD: hemodialysis; ASO: arteriosclerosis obliterans; OMI: old myocardial infarction; COPD: chronic obstructive pulmonary disease; EF: ejection fraction; PCI: percutaneous coronary intervention; LMT: left main truck

(ASO) was significantly different between the two groups. No significant differences in terms of diabetes mellitus, hypertension, dialysis, chronic obstructive pulmonary disease, and low ejection fraction rate were observed between the two groups. Meanwhile, the coronary artery stenosis was more severe in the TAR/CABG group. Most patients showed no symptoms. However, six patients complained of chest pain, whereas three had heart failure.

The operative indications were degenerative aortic arch aneurysm (219 cases), chronic dissection (26 cases), and Kommerell's diverticulum ( 1 case).

Table 2 shows the surgical data. The mean surgical time was $363.6 \pm 119 \mathrm{~min}$ in the TAR group and $472.0 \pm$ $134 \mathrm{~min}$ in the TAR/CABG group $(\mathrm{p}<0.01)$. The CPB time was $193.1 \pm 64.2 \mathrm{~min}$ and $225.5 \pm 59.6 \mathrm{~min}$ in the TAR and TAR/CABG groups, respectively $(\mathrm{p}<0.01)$. Meanwhile, the circulatory arrest time of the lower body was not different between the two groups.

The details of concomitant procedures are shown in Table 2. We performed aortic valve replacement or plasty in six cases, root replacement in four cases, and mitral valve plasty in five cases. A biatrial maze procedure for atrial fibrillation was performed in seven cases. For extensive aortic enlargement, we used elephant trunk or frozen elephant trunk techniques. The operative, CPB, and aorta cross-clamp times were greater in the TAR/CABG group than those in the TAR group. This difference might have been due to the time needed for graft harvesting, graft anastomosis, and hemorrhage stabilization.

The CABG graft design is shown in Table 3. We performed 98 distal coronary artery anastomoses (average anastomoses, 1.48). Forty-five arterial grafts (left internal thoracic artery (LITA), 39; right internal thoracic artery (RITA), 3; radial artery (RA), 2; gastroepiploic artery (GEA), 1) and 46 venous grafts were used as conduits. The LITA graft was usually used for the LAD, and the SV graft was usually used for the $\mathrm{Cx}$ artery or the RCA. Of the 39 LITA grafts, 37 were used for LAD and 2 were used for the diagonal branch. Forty-six SV grafts were used in 36 patients with 47 distal anastomoses. Nine SV grafts were used in a sequential bypass technique for the Cx and RCA.

The postoperative early outcomes are shown in Table 2. Eleven (4.5\%) in-hospital deaths occurred, and there was no 
Table 2 Surgical data

\begin{tabular}{|c|c|c|c|}
\hline & $\operatorname{TAR}(\mathrm{n}=181)$ & TAR/CABG $(n=56)$ & $\mathrm{p}$ \\
\hline Surgery time (min) & $363.6 \pm 119$ & $472.0 \pm 134$ & $<0.01$ \\
\hline $\mathrm{CPB}$ (min) & $193.1 \pm 64.2$ & $225.5 \pm 59.6$ & $<0.01$ \\
\hline Circulatory arrest (min) & $54.9 \pm 22.4$ & $59.2 \pm 18.6$ & 0.57 \\
\hline Minimum temperature $\left({ }^{\circ} \mathrm{C}\right)$ & $26.6 \pm 1.63$ & $26.6 \pm 1.57$ & 0.85 \\
\hline Bleeding (ml) & $2539 \pm 3533$ & $3398 \pm 4002$ & 0.40 \\
\hline \multicolumn{4}{|l|}{ Concomitant surgery (n) } \\
\hline MVP & 5 & 0 & \\
\hline AVR-AVP & 5 & 1 & \\
\hline Root replacement & 3 & 1 & \\
\hline Dor & 1 & 0 & \\
\hline Biatrial MAZE & 5 & 2 & \\
\hline Others & 11 & 1 & \\
\hline In-hospital mortality & $3.3 \%$ & $7.6 \%$ & 0.14 \\
\hline Intubation hours (h) & $5.2 \pm 0.9$ & $5.4 \pm 1.0$ & 0.91 \\
\hline Reintubation & $6.6 \%$ & $12.3 \%$ & 0.15 \\
\hline Tracheotomy & $5.5 \%$ & $7.7 \%$ & 0.53 \\
\hline Peak CK & $1164 \pm 11998$ & $1109 \pm 1135$ & 0.49 \\
\hline Peak CK-MB & $16.8 \pm 19.0$ & $39.6 \pm 176$ & 0.003 \\
\hline ICU stay (days) & 5.0 & 7.3 & 0.24 \\
\hline Hospital stay & 22.0 & 24.3 & 0.48 \\
\hline \multicolumn{4}{|l|}{ Complications } \\
\hline Pneumonia & $8.8 \%$ & $13.8 \%$ & 0.28 \\
\hline Stroke & $5.5 \%(10)$ & $7.7 \%(5)$ & 0.53 \\
\hline Mediastinitis & $1.1 \%$ & $1.5 \%$ & 0.80 \\
\hline PMI & $0 \%$ & $0 \%$ & - \\
\hline
\end{tabular}

TAR: total aortic arch replacement; CABG: coronary artery bypass grafting;

CPB: cardio-pulmonary bypass; MVP: mitral valve plasty; AVR: aortic valve replacement; AVP: aortic valve plasty; CK: creatine kinase; ICU: intensive care unit; PMI: perioperative myocardial infarction

Table 3 Details of coronary artery bypass surgery and early patency

\begin{tabular}{lcc}
\hline \multicolumn{3}{c}{ TAR/CABG group } \\
\hline Anastomosis & 98 (average, 1.48) & \\
Artery graft & 45 conduits (LITA 39, RITA 3, RA 2, GEA 1) & \\
Vein graft & 46 conduits & \\
LAD & Total: 37 (LITA: 37) & \\
Dx & Total: 4 (LITA: 2, SV: 2) & \\
Cx & Total: 26 (SV: 23, RITA: 2, RA: 1) & \\
RCA & Total: 31 (SV: 29, RA: 1, GEA: 1) & \\
\hline & Evaluation (evaluation/total) & $64 \%$ \\
\hline LITA & $33 / 37$ & $100 \%$ \\
RITA & $2 / 3$ & $95 \%$ \\
RA & $2 / 2$ & Early patency \\
SV & $39 / 44$ & $94 \%$ \\
\hline
\end{tabular}

TAR: total aortic arch replacement; CABG: coronary artery bypass grafting; LITA: left internal thoracic artery; RITA: right internal thoracic artery; RA: radial artery; GEA: gastroepiploic artery; LAD: left anterior descending artery; Dx: diagonal branch; Cx: circumflex; RCA: right coronary artery; SV: saphenous vein

significant difference between the TAR and TAR/CABG groups. The incidence of postoperative infectious pneumonia stroke, and deep sternal wound infection was not significantly different. Intubation hours were also not significantly different. Perioperative myocardial infarction was not observed in any cases.

The long-term mortality data are shown in Fig. 1A. The mean clinical follow-up period was $47.5 \pm 37.8$ months, and 

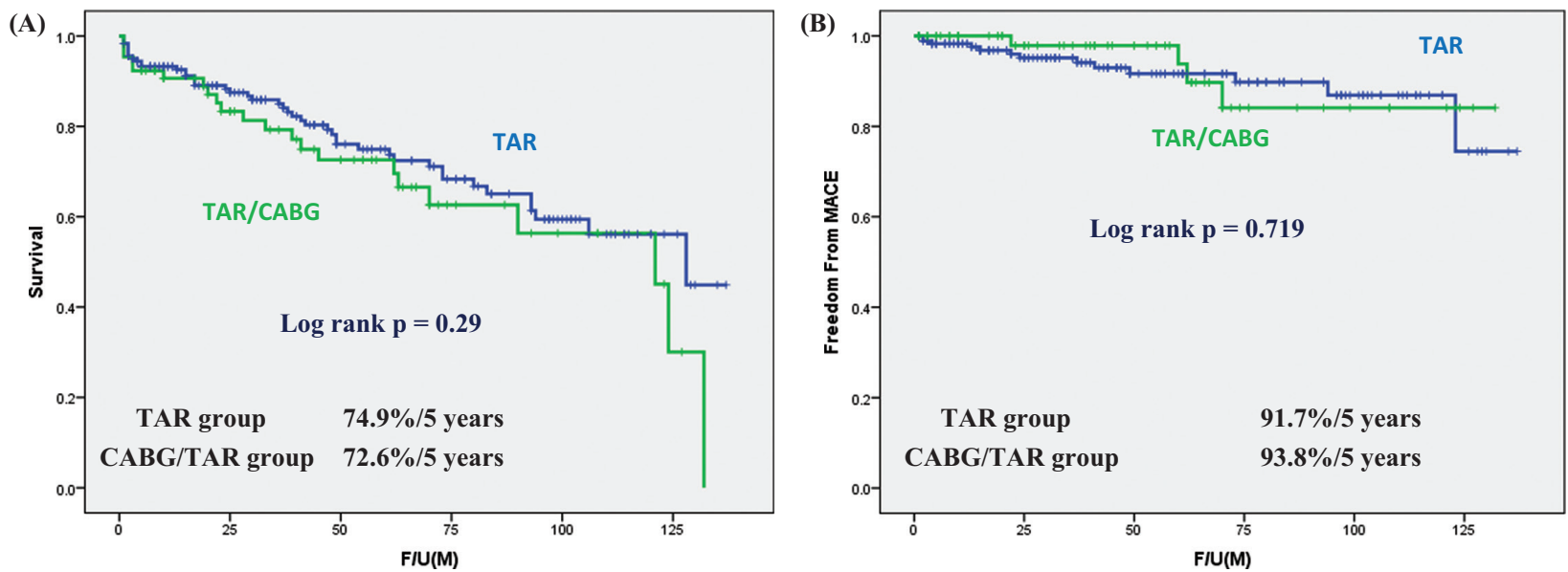

Fig. 1 (A) Survival curve. (B) Freedom from MACE. TAR: total aortic arch replacement; CABG: coronary artery bypass grafting; MACE: major adverse cardiovascular events

Table 4 Risk factors for long-term mortality identified by univariate and multivariate analysis

\begin{tabular}{lcccc}
\hline \multirow{2}{*}{ Variables } & \multicolumn{2}{c}{ Univariate } & \multicolumn{2}{c}{ Multivariate } \\
\cline { 2 - 5 } & OR $(95 \% \mathrm{CI})$ & $\mathrm{p}$ value & OR $(95 \% \mathrm{CI})$ & $\mathrm{p}$ value \\
\hline Age & $1.04-1.12$ & $<0.01$ & $1.04-1.13$ & $<0.01$ \\
Sex & $0.69-1.23$ & 0.53 & & \\
IHD & $1.18-3.11$ & 0.008 & $1.10-3.76$ & 0.02 \\
OMI & $0.79-3.25$ & 0.19 & & 0.76 \\
CABG & $0.79-2.20$ & 0.29 & $0.47-1.74$ & \\
CHF & $0.77-1.94$ & 0.39 & & \\
Stroke & $0.77-1.94$ & 0.50 & & \\
eGFR & $1.25-3.77$ & 0.06 & & \\
ASO & $0.76-4.12$ & 0.19 & $1.00-3.01$ & \\
DM & $0.59-2.87$ & 0.53 & & \\
COPD & $0.87-3.44$ & 0.11 & & \\
Smoking & $0.44-1.43$ & 0.79 & & \\
\hline
\end{tabular}

OR: odds ratio; CI: confidence interval; IHD: ischemic heart disease; OMI: old myocardial infarction; $\mathrm{CABG}$ : coronary artery bypass grafting; $\mathrm{CHF}$ : congestive haert failure; eGFR: estimated glemerular filtration rate; ASO: arteriosclerosis obliterans; DM: diabetes mellitus; COPD: chronic obstructive pulmonary disease

the follow-up rate was $93.1 \%$. Freedom from all-cause death was $74.9 \%$ (TAR) and $72.6 \%$ (TAR/CABG) after 5 years (log-rank, $\mathrm{p}=0.29$ ). Freedom from MACE was 91.7\% (TAR) and 93.8\% (TAR/CABG) after 5 years (logrank, $\mathrm{p}=0.72)($ Fig. 1B). Postoperative needs for percutaneous coronary intervention (PCI) occurred in one case in the TAR group and three cases in the TAR/CABG group. The case in the TAR group had non-ischemic heart disease, but this case underwent PCI to LAD in acute myocardial infarction after 9 years. In contrast, two patients in the TAR/CABG group whose saphenous vein (SV) grafts were patent as confirmed by early postoperative $\mathrm{CT}$ cardiac images showed the graft occluded during the preoperative examination of the abdominal aortic aneurysm. They did not have chest pain but had previously undergone PCI. The other postoperative PCI case in the TAR/CABG group, a 76-year-old man, was hospitalized for heart failure after 5 years. Coronary angiography (CAG) showed patency of LITA-LAD grafting but SV graft occlusion. PCI was performed for $\mathrm{Cx}$ and RCA.

Univariate analysis showed that preoperative age, ischemic heart disease, and estimated glemerular filtration rate (eGFR) were all predictors of long-term mortality. Multivariate analysis showed that age, ischemic heart disease, and eGFR were also predictors of long-term mortality. Concomitant TAR with CABG was not a risk factor for long-term mortality (Table 4). 


\section{Discussion}

The procedure undertaken for TAR is quite complex and requires hypothermia for brain protection. The surgery and CPB times are longer than those for other types of cardiovascular surgery, and there is always a risk of postoperative complications. Kazui et al. reported the surgical outcomes of 220 consecutive patients who underwent total arch replacement and found that the overall in-hospital mortality rate was $12.7 \% .^{9)}$ However, more recently, surgical outcomes have been improving. Kulik et al. reported excellent surgical outcomes for TAR, with a 30-day mortality rate of $5.7 \% .^{2}$

The Japanese population is rapidly aging, and consequently, patients are increasingly older. To maintain good surgical outcomes for aortic surgery in these high-risk patients, better knowledge of intensive care and improved surgical skills are required. Moreover, some patients not only have aortic arch aneurysms but also have significant coronary artery stenosis. Some reports have described concomitant procedures, such as TAR with CABG, as leading to increased morbidity and mortality. ${ }^{4-5)}$ Kuniyoshi et al. reported that CABG involving three or more vessels combined with aortic arch replacement may increase the operative risk. ${ }^{5)}$ However, other studies have reported that performing concomitant procedures is perfectly safe. ${ }^{6-7)}$ Because of these discrepancies in the literature, the performance of concomitant procedures remains controversial.

In our study, some patients who underwent CABG were asymptomatic; however, no cases of perioperative myocardial ischemic events were observed, and concomitant TAR with CABG did not increase postoperative morbidities and mortality. Multivariate analysis showed that concomitant TAR with CABG was not a risk factor for long-term mortality, although ischemic heart disease (IHD) was a risk factor. On the basis of these results and the fact that $11.2 \%$ of patients had a history of PCI in the TAR group, concomitant TAR with CABG should be performed if patients have severe coronary artery stenosis.

In contrast, some papers have reported that smoking, $\mathrm{CPB}$ time, perioperative bleeding volume, transfusion, and recurrent nerve injury are risk factors for pulmonary dysfunction after cardiac surgery. ${ }^{5,10-11)}$ Our study showed that prolonged operative time, $\mathrm{CPB}$ time, and high creatine phosphokinase-MB isozyme (CK-MB) scores were risk factors. There was no significant difference in the amount of bleeding. Yokoyama et al. reported off-pump CABG can minimize the myocardial ischemic time to decrease the risk of postoperative myocardial failure.4)
However, we speculate that this method increases the complexity to some degree, when to perform distal anastomosis in $\mathrm{CABG}$, one of the important factors. In our study, distal anastomosis, including LITA-LAD anastomosis was performed during core cooling under on-pump beating. In addition, CABG was performed on key coronary vessels. We believe that because the number of symptomatic patients who underwent postoperative PCI was less, our CABG strategy was acceptable.

Graft design in concomitant TAR with CABG is an important issue. When undergoing TAR, the use of LITA is controversial. Yokoyama et al. reported that the use of LITA affected hypoperfusion and spasm caused by bleeding during the reconstruction of the left subclavian artery. ${ }^{4}$ However, the use of LITA-LAD has been a gold standard in isolated CABG. Similarly, patients who receive LITALAD grafting for aortic valve replacement improved longterm mortality in comparison with patients who receive only an SV graft. ${ }^{12)}$ Some recent studies report the use of LITA-LAD with a good patency rate in this setting. ${ }^{6,13)} \mathrm{We}$ also performed LITA-LAD, and the rest of the anastomosis was often used as an SV graft to improve convenience and reduce the surgical time. Our results showed that LITA-LAD had been used in all cases. Postoperative PCI in the TAR/CABG group was performed for three cases because of SV graft occlusion, despite a patent LITALAD. On the other hand, the use of RITA is found only in few cases. There is a need for verification. But if LITALAD was performed good patency, RITA might not always necessary because no significant difference was seen in the long-term outcomes of the two groups.

Our study has several limitations. This was a retrospective study from a single center. It was not randomized, and the number of patients was small. Furthermore, some patients had undergone other concomitant surgeries that were not TAR and CABG. We were unable to follow-up some of the patients, and in some cases, the follow-up period was insufficient.

\section{Conclusion}

The mortality of TAR in our institution was equivalent to or better than that of other institutions. The surgery and $\mathrm{CPB}$ times were longer in the TAR/CABG group; however, this did not increase postoperative morbidities in this group, and there were no patients with myocardial ischemia. Moreover, no significant difference was seen in the long-term outcomes of the two groups. On the basis of these findings, $\mathrm{CABG}$ should be performed for patients 
who have an aortic aneurysm with significant coronary artery stenosis without an additional risk.

\section{Disclosure Statement}

The authors have declared no conflicts of interest. None of the contributors has no disclose interests with regard to commercial support.

\section{References}

1) Kazui T, Yamashita K, Washiyama N, et al. Aortic arch replacement using selective cerebral perfusion. Ann Thorac Surg 2007; 83: S796-8; discussion S824-31.

2) Kulik A, Castner CF, Kouchoukos NT. Outcomes after total aortic arch replacement with right axillary artery cannulation and a presewn multibranched graft. Ann Thorac Surg 2011; 92: 889-97.

3) Okada K, Omura A, Kano H, et al. Short and midterm outcomes of elective total aortic arch replacement combined with coronary artery bypass grafting. Ann Thorac Surg 2012; 94: 530-6.

4) Yokoyama H, Sato Y, Takase S, et al. Introduction of off-pump coronary artery bypass into aortic arch aneurysm repair: a new solution for the surgical treatment of multiorgan arteriosclerosis. J Thorac Cardiocvasc Surg 2005; 129: 935-6.

5) Kuniyoshi Y, Koja K, Miyagi K, et al. Surgical treatment of aortic arch aneurysm combined with coronary artery stenosis. Ann Thorac Cardiovasc Surg 2002; 8: 369-73.

6) Okada K, Omura A, Kano H, et al. Recent advancements of total aortic arch replacement. J Thorac Cardiovasc Surg 2012; 144: 139-45.

7) Takashima N, Suzuki T, Asai T, et al. Outcome of total arch replacement with coronary artery bypass grafting. Eur J Cardiothorac Surg 2015; 47: 990-4.

8) Tamura N, Komiya T, Sakaguchi G, et al. 'Turn-up' anastomotic technique for acute aortic dissection. Eur J Cardiothorac Surg 2007; 31: 548-9.

9) Kazui T, Washiyama N, Muhammad BA, et al. Total arch replacement using aortic arch branched grafts with the aid of antegrade selective cerebral perfusion. Ann Thorac Surg 2000; 70: 3-8; discussion 8-9.

10) Ueda T, Shimizu H, Shin $H$, et al. Detection and management of concomitant coronary artery disease in patients undergoing thoracic aortic surgery. Jpn J Thorac Cardiovasc Surg 2001; 49: 424-30.

11) Leal-Noval SR, Rincón-Ferrari MD, García-Curiel A, et al. Transfusion of blood components and postoperative infection in patients undergoing cardiac surgery. Chest 2001; 119: 1461-8.

12) Gall S, Lowe JE, Wolfe WG, et al. Efficacy of the internal mammary artery in combined aortic valve replacementcoronary artery bypass grafting. Ann Thorac Surg 2000; 69: 524-30.

13) Fukui T, Shimokawa T, Tabata M, et al. Outcomes of total aortic arch replacement with coronary artery bypass grafting. Interact Cardiovasc Thorac Surg 2011; 13: $284-7$. 